\title{
El efecto de la traducción pedagógica en la precisión morfosintáctica: un estudio preliminar con estudiantes avanzados de ESL
}

Mag. Laura Gasca Jiménez ${ }^{1}$, Universidad de Houston, Estados Unidos

Recibido: $\quad 6$ de abril, 2017

Aceptado: 23 de agosto, 2017

\section{Resumen}

En este artículo se exponen los resultados de un estudio longitudinal de cuatro semanas de duración sobre el efecto de la traducción pedagógica en la precisión morfosintáctica en la segunda lengua. Seis estudiantes universitarios matriculados en un curso de nivel avanzado de español como segunda lengua en la Universidad de Houston participaron en el estudio. El curso tenía como objetivo contribuir al desarrollo de la competencia comunicativa de los estudiantes mediante la introducción de la práctica de la traducción directa ( $L 2>L 1$ ) e inversa ( $L 1>L 2)$, con un particular énfasis en esta última modalidad. Los participantes llevaron a cabo una actividad de traducción a modo de pretest/postest al comienzo y al final del curso. Los resultados sugieren que la incorporación de la traducción pedagógica mediante una metodología activa que promueve la reflexión y combina el trabajo individual y colaborativo tiene un impacto considerable en la precisión morfosintáctica en la segunda lengua. Este impacto positivo se hace particularmente notorio en el uso de preposiciones, adjetivos y del tiempo y modo verbales. Así, este estudio se suma a los trabajos de Cook (2010) y González Davies (2002), que proponen la práctica de la traducción inversa (L1>L2) para el desarrollo de la precisión gramatical.

\section{Abstract \\ The Effect of Pedagogic Translation on Morphosyntactic Accuracy: A Preliminary Study with Advanced SSL Students.}

This article presents the results of a longitudinal four-week study about the effect of pedagogic translation on morphosyntactic accuracy in a second language. Six undergraduate students enrolled in an advanced Spanish as a second language course at the University of Houston participated in the study. The main objective of this course was to continue developing the students' communicative competence through the introduction of direct $(\mathrm{L} 2>\mathrm{L} 1)$ and reverse $(\mathrm{L} 1>\mathrm{L} 2)$ translation activities, with an emphasis on the latter. The participants completed a pre and post-test translation activity at the beginning and at the end of the course. The results suggest that the incorporation of pedagogic translation within an active methodology that promotes reflection and combines individual and collaborative work has a significant impact on morphosyntactic accuracy in the target language. This positive impact is particularly noticeable in the use of prepositions, adjectives, and verbal tense and mood. This study contributes to the work of Cook (2010) and González Davies (2002), who propose the practice of reverse translation $(\mathrm{L} 1>\mathrm{L} 2)$ for the development of grammatical accuracy.

1 Laura Gasca es bachiller en Inglés, graduada de la Universidad Complutense de Madrid, España, Magíster en Traducción Española de la Universidad Kent State, en Ohio, Estados Unidos y doctoranda de Lingüística Española de la Universidad de Houston, Texas, Estados Unidos. En esta misma casa de enseñanza superior se desempeña como docente e investigadora. Contacto: Igascajimenez@uh.edu

Laura Gasca Jiménez. El efecto de la traducción pedagógica en la precisión morfosintáctica: un estudio preliminar con estudiantes avanzados de ESL. Revista Comunicación. Año 38, volumen 26, número 2, julio-diciembre, 2017. Instituto Tecnológico de Costa Rica. ISSN: 0379-3974 / e-ISSN1659-3820.
PALABRAS CLAVE:

traducción pedagógica, precisión morfosintáctica, traducción inversa, preposiciones, adjetivo.

\section{KEY WORDS:}

pedagogic translation, morphosyntactic accuracy, reverse translation, prepositions, adjective. 


\section{INTRODUCCIÓN}

La traducción con fines didácticos está siendo redefinida como una actividad comunicativa con el potencial de facilitar la enseñanza y adquisición de lenguas. Los argumentos teóricos que promueven su integración son numerosos (véase Carreres y Noriega-Sánchez, 2011; Cook, 2010; González Davies, 2004; Colina, 2002; Malmkjaer, 1998 y Duff, 1989, entre otros). No obstante, los estudios empíricos que evalúan su potencial como herramienta didáctica son muy escasos (véase Källkvist, 2004, 2008 y Sánchez Cuadrado, 2016).

Este estudio se enmarca dentro de un proyecto de investigación más amplio que tiene como objetivo investigar el potencial didáctico de la traducción pedagógica en la enseñanza de lenguas. En un estudio previo ${ }^{2}$ se investigó el impacto general de la traducción pedagógica en el desarrollo de habilidades lingüísticas y extralingüísticas en estudiantes avanzados de español como segunda lengua. Los resultados de dicha investigación ponen de manifiesto que la incorporación de la traducción pedagógica mediante una metodología activa y colaborativa que promueve la reflexión tiene un impacto significativo en el desarrollo de habilidades lingüísticas en la segunda lengua, particularmente en la habilidad de transferir información en actividades de traducción inversa, desde la primera lengua a la segunda lengua (L1>L2) y en la habilidad de usar técnicas de traducción con mayores niveles de precisión. Los resultados también indican que la incorporación de la traducción mediante esta metodología permite que los estudiantes traten la traducción como una actividad comunicativa definida por factores tanto lingüísticos como extralingüísticos y desarrollen las habilidades de identificar y respetar los rasgos diferenciales entre sus dos lenguas.

El presente trabajo constituye una ampliación de dicho estudio y tiene como objetivo investigar el impacto de la traducción en el perfeccionamiento de habilidades morfosintácticas en la segunda lengua. Los resultados sugieren que la práctica de la traducción inversa (L1>L2) contribuye positi-

2 Gasca Jiménez, L. (en prensa). Las posibilidades de la traducción pedagógica para la enseñanza del español como segunda lengua. Estudios de Lingüística Aplicada, 66. vamente a la precisión morfosintáctica. A pesar de ser un estudio de carácter preliminar, por contar con un número reducido de participantes y basarse en el análisis de un número limitado de muestras de escritura, la presente investigación es de suma relevancia para el campo de la traducción pedagógica, ya que valida empíricamente su valor didáctico en la enseñanza de segundas lenguas.

El artículo está dividido en seis partes: en la primera se presentan los principales postulados teóricos en relación con la traducción pedagógica, en la segunda se incluyen los estudios empíricos previos que se han realizado siguiendo esta línea de investigación, en la tercera se explica la metodología empleada, en la cuarta se describen y analizan los resultados obtenidos, en la quinta se dan las conclusiones y se proponen implicaciones pedagógicas a partir de estas y en la sexta, por último, se exponen las limitaciones del estudio.

\section{MARCO TEÓRICO}

Desde el ya abandonado método de enseñanza gramática-traducción, la traducción ha sido ignorada como una posible herramienta didáctica. Como afirma el reconocido lingüista Guy Cook (2010), la noción de que la traducción no es beneficiosa para la adquisición de lenguas está tan generalizada que apenas se ha investigado. Además de ser relegada al exilio, la traducción ha sido despreciada por considerarse una actividad extremadamente compleja y laboriosa, independiente de las cuatro destrezas que forman la competencia lingüística que, además de entorpecer el aprendizaje de una segunda lengua (en adelante L2), promueve la transferencia negativa ${ }^{3}$ desde la lengua materna (en adelante L1). Alan Maley recoge perfectamente estos sentimientos en el prólogo al libro titulado Translation del lingüista, profesor y traductor Alan Duff publicado en 1989: 
Translation has long languished as a poor relation in the family of language teaching techniques. It has been denigrated as 'uncommunicative', 'boring', 'pointless', 'difficult', 'irrelevant', and the like, and has suffered from too close an association with its cousin, Grammar. Along with its other traditional cousins Literature, Dictation, Vocabulary, Reading Aloud, etc., it has been pushed into the methodological lumber room (p. 3).

Muchos autores coinciden en que esta reputación negativa de la traducción se debe al desechado método gramática-traducción (Malmkjaer, 1998; González, 2004 y Cook, 2010, entre otros). El objetivo de este método era facilitar la lectura de literatura en la lengua meta, por lo que se trabajaban exclusivamente la comprensión lectora y la producción escrita. Las reglas gramaticales se analizaban y memorizaban para luego traducir oraciones desde y hacia la lengua meta ${ }^{4}$. Con la llegada del método directo y el consecuente énfasis en la lengua hablada, la traducción fue expulsada de las clases de lengua para dar paso a lo que Cummins (2008) denomina el principio del monolingüismo, según el cual la primera lengua y la segunda se mantienen estrictamente separadas la una de la otra durante el aprendizaje.

Los argumentos más frecuentes a los que han recurrido varios autores como Lado (1964) y Gatenby (1967) para negar la utilidad de la traducción son que no es un proceso natural, que crea una falsa ilusión de equivalencia entre las dos lenguas y promueve la transferencia negativa desde la L1. Lado (1964) sostiene que la traducción no permite la práctica en una L2, ya que crea una falsa ilusión de equivalencia que compromete la precisión gramatical y promueve las traducciones literales agramaticales. Además, va más allá al afirmar que la traducción es un proceso complejo e innecesario para desarrollar la expresión oral, la expresión escrita, la comprensión auditiva y la comprensión lectora. En definitiva, este autor concibe la traducción como una actividad contraproducente, a menos que se domine

$4 \quad$ Véase Richards y Rodgers (2014) para una explicación más detallada. una L2: "Good translation cannot be achieved without mastery of a second language. We, therefore, teach the language first, and then we may teach translation as a separate skill, if that is considered desirable" (p. 54). Para Gatenby (1967) la traducción es una actividad artificial y contraproducente que debería evitarse a toda costa como actividad y como herramienta de evaluación. Al igual que Lado (1964), desecha por completo el potencial didáctico de la traducción, relegándola a una destreza que depende del dominio de dos lenguas:

Translation is a deceptive process in that, being laborious, it persuades teacher and pupil that a great deal has been accomplished. Unfortunately, such work is all but useless. Translation may give meaning, but it does not teach. It perpetuates the time-wasting habit of always associating the new language with the old, and it hinders full comprehension. As a skill, the proper time for practicing it is when an equal command of both languages has been obtained (p. 70).

En las últimas décadas, se han propuesto numerosos argumentos para reivindicar la rehabilitación de la traducción con fines didácticos dentro de marcos de enseñanza comunicativos y metodologías activas. Cook (2010) y González Davies (2002) enfatizan el desarrollo de la precisión gramatical, particularmente mediante la práctica de la traducción inversa (L1>L2). Según estos autores, la práctica de la traducción, a diferencia de la escritura libre, obliga a los estudiantes a enfrentarse a dificultades en su segunda lengua:

Let's think about what happens when students write a composition, for instance: They can avoid problematic areas and communicate effectively using avoidance strategies. With translation they have to relay what is in the source text, whether they are in command of the linguistic and extra- linguistic knowledge necessary to do so or not (González Davies, 2002, p. 70).

Cook (2010) reconoce la importancia de desarrollar la habilidad de parafrasear mediante 
actividades abiertas, pero enfatiza la utilidad de combinar dichas actividades con ejercicios de traducción que permitan centrarse en dificultades específicas y vacíos de conocimiento:

Close translation not only reveals problems, however, it can also be used proactively, in order to deliberately draw students' attention to difficult aspects of the new language. For the bilingual teacher or materials writer who already knows their students' language and the problems which are likely to emerge from them in the new language, it is possible to design close translation activities which will concentrate attention to those problem areas (p. 138).

Varios autores han enfatizado que la traducción permite controlar la transferencia negativa y fomentar la transferencia positiva. Snell-Hornby (1985), basándose en su experiencia como profesora de un curso universitario de traducción alemán-inglés, se opone al rechazo absoluto de la traducción en todas las etapas de adquisición de una segunda lengua. Esta autora sugiere que la práctica de la traducción permite explorar las relaciones interlingüísticas y contribuir positivamente al perfeccionamiento de la precisión gramatical. Siguiendo una línea muy similar, Titford (1985) argumenta que las actividades de traducción constituyen el contexto idóneo para explicar la interferencia negativa desde la L1 sin la necesidad de recurrir a largas explicaciones de corte metalingüístico. Sørensen (1990) enfatiza el valor didáctico de comparar y contrastar las dos lenguas de un aprendiente mediante la práctica directa e inversa de la traducción, particularmente en el caso de estudiantes de nivel avanzado: "This activity makes advanced students aware of tricky dissimilarities and incongruities between languages" (p. 202). Por su parte, Harvey (1996) destaca la utilidad de la traducción para hacer evidentes los errores que pueden ser el resultado de una superposición inconsciente de las estructuras de la lengua materna. Malmkjær (1998) reitera, por un lado, que la traducción contribuye a desarrollar la habilidad de relacionar dos sistemas lingüísticos, lo que permite -a su vez- minimizar la inter- ferencia negativa y maximizar la positiva. Por otro lado, afirma que es innegable que la traducción crea interferencia, pero afirma que la práctica de la traducción pone en evidencia dicha interferencia y permite desarrollar mecanismos de control para limitarla.

En definitiva, a pesar de las preconcepciones existentes sobre la traducción como consecuencia del método gramática-traducción, numerosos autores apuntan que es una actividad comunicativa con el potencial de contribuir al perfeccionamiento de la precisión gramatical y a la limitación de la transferencia negativa.

\section{ESTUDIOS PREVIOS}

Es conveniente enfatizar que la traducción pedagógica es un campo de investigación abierto, dado que son pocas las investigaciones empíricas que se han realizado hasta el momento para validar su aplicación (Carreres y Noriega-Sánchez, 2011; Cummins, 2007; Hurtado Albir, 2001 y KäIlkvist, 2008). Se han Ilevado a cabo varios estudios empíricos que investigan su potencial como herramienta de evaluación (véase Kobayashi y Rinnert, 1992; Källkvist, 1996; Cohen y BrooksCarson, 2001 y Schjoldager, 2004, entre otros). No obstante, son pocos los estudios empíricos que han examinado los efectos que tiene como herramienta didáctica (véase Snellings Van Gelderen y De Glopper, 2002; Källkvist, 2004, 2008; Vaezi y Mirzaei, 2007; Laufer y Girsai, 2008; Belpoliti y Plascencia-Vela, 2013 y Sánchez Cuadrado, 2016). A continuación, se presentan dos de dichas investigaciones que examinan específicamente el impacto de la traducción en el desarrollo de habilidades morfosintácticas.

Källkvist (2004) investiga la posibilidad de perfeccionar el uso de estructuras morfosintácticas en una L2 mediante la práctica de la traducción. Un total de 44 universitarios hablantes nativos de sueco participaron en este estudio de trece semanas de duración. Los participantes se dividieron en tres grupos, dos experimentales y uno no experimental. El primer grupo experimental (el grupo de traducción) realizó consistentemente ejercicios de traducción para trabajar estructuras morfosintácticas específicas, mientras que el 
segundo (el grupo de no traducción) cubrió las mismas estructuras, pero mediante ejercicios en la L2. El grupo no experimental estaba formado por estudiantes de último año que no recibían una instrucción explícita de gramática en la L2, pero sí una exposición extensa a la lengua mediante la lectura y la escritura. Todos los sujetos completaron un pretest que constaba de tres tareas: una actividad de opción múltiple, una actividad de traducción y una actividad de reescritura. Al final del periodo de instrucción, todos los sujetos volvieron a completar las mismas tareas. Además del pretest y postest, todos hicieron una prueba más breve durante la tercera semana de instrucción que constaba de un ejercicio de traducción y uno de rellenar huecos. Esta prueba tenía como objetivo evaluar la adquisición de una estructura gramatical estudiada (el uso del artículo definido).

Los resultados de esta investigación se limitan al análisis de dicha prueba. Por el número limitado de ítems (3), los resultados deben interpretarse como provisionales. Este estudio muestra que, en cuanto al uso del artículo definido respecta, los estudiantes con una motivación alta que son expuestos a ejercicios en la L2 pueden desempeñarse igual de bien o incluso mejor en un ejercicio de traducción que los estudiantes que habían estado expuestos a esta. Los resultados generales evidencian que los dos grupos experimentales obtuvieron resultados similares, mientras que el grupo de no gramática tuvo resultados considerablemente inferiores. La autora recomienda combinar ejercicios de traducción con otros en la L2 que permitan centrarse en la forma.

Källkvist (2008) retoma los datos de su estudio preliminar del 2004 para comparar de una forma más minuciosa los efectos de ejercicios de traducción inversos (L1 $>$ L2) y ejercicios en la L2 (de rellenar huecos y de transformación) a través de las trece semanas de instrucción. El análisis de los pretests y los postests muestra que no hay una diferencia estadísticamente significativa entre los resultados de los dos grupos en los ejercicios de elección múltiple y traducción. Sin embargo, la autora destaca que en el ejercicio de traducción el grupo de traducción obtuvo resultados que se aproximan al nivel de significancia estadística y conjetura que con un grupo mayor de participantes y un número mayor de ítems en el ejercicio de traducción las diferencias observadas entre los dos grupos en dicho ejercicio hubieran sido estadísticamente significativas. Los resultados del estudio sugieren que los estudiantes avanzados de L2 pueden beneficiarse de la traducción, pero no como única forma de instrucción, sino en combinación con otros ejercicios en la L2.

\section{METODOLOGÍA}

Enmarcado dentro de la misma línea de investigación que los estudios presentados en la sección previa, el presente estudio contribuye a la investigación sobre los efectos de la traducción pedagógica en la precisión lingüística, área que como ya se ha indicado-adolece de más estudios cuantitativos.

\section{Preguntas de investigación}

Las preguntas específicas que este estudio pretende responder son las siguientes:

a) ¿Hay un aumento de errores morfosintácticos tras el periodo de instrucción?

b) ¿Hay una disminución de errores morfosintácticos tras el periodo de instrucción?

c) ¿Qué categorías morfosintácticas se ven afectadas positiva o negativamente tras el periodo de instrucción?

\section{Participantes}

Los participantes fueron ocho estudiantes de español matriculados en un curso avanzado de español dirigido a estudiantes de licenciatura en la Universidad de Houston. El curso forma parte de la especialización en español a la que los estudiantes acceden opcionalmente tras completar satisfactoriamente dos cursos básicos y dos cursos intermedios de español como segunda lengua. En el momento en el que se llevó a cabo el estudio, los participantes se encontraban en una estancia en el extranjero en Cádiz, España. Dos estudiantes fueron descartados por no haber estado presentes durante la administración de todos los instrumentos. Así, el total de participantes se redujo a 6 (véase la Tabla 1 ). 
Tabla 1: Características de los participantes

\begin{tabular}{ccccc}
\hline Ref. Género & $\begin{array}{c}\text { Instrucción } \\
\text { en español } \\
\text { (educación } \\
\text { primaria) }\end{array}$ & $\begin{array}{c}\text { Instrucción } \\
\text { en español } \\
\text { (educación } \\
\text { secundaria) }\end{array}$ & $\begin{array}{c}\text { Instrucción } \\
\text { en español } \\
\text { (nivel uni- } \\
\text { versitario) }\end{array}$ \\
\hline P1 & M & 2 años & 3 años & 3 semestres \\
P3 & F & 1 año & 4 años & 4 semestres \\
P4 & F & 3 años & 1 año & 2 semestres \\
P5 & F & - & 2 años & 5 semestres \\
P6 & F & 2 años & 3 años & 1 semestre \\
P7 & F & 3 años & 2 años & 3 semestres \\
\hline
\end{tabular}

Instrucción

El objetivo general del curso fue contribuir al desarrollo de la competencia comunicativa de los estudiantes mediante la introducción de la práctica de la traducción directa (de español a inglés) e inversa (de inglés a español), con un particular énfasis en esta última modalidad. El carácter del curso fue intensivo, con cuatro encuentros semanales (de lunes a jueves) de dos horas de duración durante cuatro semanas consecutivas. Siguiendo las propuestas de Kiraly (2000) y González Davies (2004), desde la didáctica de la traducción se favoreció una metodología activa, centrada en el estudiante, en la que se priorizó el proceso (la práctica de la traducción) sobre el producto (el texto traducido).

Durante las primeras sesiones se presentaron diferentes fuentes de consulta (diccionarios bilingües, monolingües, corpora, etc.) que permitieron que los estudiantes se enfrentaran a la traducción como una actividad interpretativa que depende de factores extralingüísticos, como por ejemplo el objetivo del texto y la audiencia meta a la que va dirigido. El resto de las sesiones se enfocaron en la práctica colaborativa de tareas de traducción, así como la reflexión grupal sobre las características de los textos, los problemas de traducción, las posibles soluciones, etc. A partir de dichas actividades, se introdujo progresivamente terminología relacionada con la práctica profesional de la traducción. Se presentaron diferentes técnicas de traducción, se analizaron los rasgos diferenciales principales entre el inglés y el español a nivel sintáctico, morfológico y de puntuación y por último, se exploró la dimensión social de la traducción y la importancia de considerar las variedades lingüísticas del español al traducir. Además de las actividades colaborativas en clase, cada semana los estudiantes tuvieron que trabajar de forma individual en un encargo de traducción acompañado de una breve reflexión sobre las características del texto, las principales dificultades a las que se enfrentaron y las soluciones que pusieron en práctica (véase Anexo A para un ejemplo de tarea y entrega).

\section{Instrumentos}

Se empleó una actividad de traducción inversa (L1>L2) como pretest/postest para evaluar el impacto del periodo de instrucción (véase Anexo B). El texto de origen de la actividad de traducción es un fragmento de 160 palabras de una noticia publicada en inglés en la página web Yahoo News sobre el lanzamiento de un nuevo producto de Apple. Se eligió por tratarse de un tema sencillo de actualidad, pero que por su terminología semitécnica fomentaría el uso de técnicas de traducción.

\section{Procedimiento}

Los participantes realizaron la actividad de traducción a modo de pretest/postest durante el primer y el último día del curso. Tuvieron una hora de duración para completarla y no se les permitió usar materiales de consulta. A lo largo del curso, no tuvieron acceso a la actividad y no se les informó que volverían a realizarla al finalizar este, como recomiendan Mellinger y Hanson (2017) para garantizar la validez de los resultados en investigaciones que hacen uso de este procedimiento.

Partiendo de la técnica del análisis de errores (James, 1998) y otros estudios que han Ilevado a cabo análisis similares con estudiantes de español (Fairclough y Mrak, 2003), se creó una clasificación de errores que permitió cuantificar los errores morfosintácticos localizados en las traducciones. Se establecieron nueve categorías de errores: concordancia dentro de la frase nominal $(\mathrm{CN})$, concordancia entre el sujeto y el verbo $(\mathrm{CV})$, tiempo o modo (TM), preposiciones $(\mathrm{PR})$, adverbios (ADV), clíticos (CL), adjetivos (ADJ), ser o estar (SE) y otros (OT). Dichas categorías se subcategorizaron en cinco tipos de error: omisión, adición, sustitución, orden y combinación ${ }^{5}$. Los errores localizados en las traducciones se cuantificaron y se clasificaron

5 Se refiere a la combinación de dos formas que tiene como resultado una combinación inexistente en la lengua en cuestión. 
siguiendo esta clasificación. Para calcular el número total de palabras producidas durante los dos periodos de instrucción, se sumó el total de palabras, excluyendo comentarios en inglés de los participantes sobre la elección o elisión de expresiones.

\section{RESULTADOS Y DISCUSIÓN}

El número total de palabras en el pretest fue 897; de las cuales, 125 se codificaron como errores $(0,13 \%)$. En el postest, el número de palabras ascendió a 936 y el número total de errores disminuyó a $89(0,09 \%)$, como se puede ver en la Tabla 2. Las categorías lingüísticas con la mayor frecuencia de errores durante los dos periodos de evaluación fueron: TM (Pre $=0,30$; Post $=0,28$ ), $\mathrm{CN}(\mathrm{Pre}=0,22 ;$ Post $=0,24), \mathrm{PR}(\mathrm{Pre}=0,22 ;$ Post $=$ $0,16)$ y $\mathrm{ADJ}($ Pre $=0,12 ;$ Post $=0,07)$. El número de errores en las categorías de CV, ADV, CL, SE y OT fue muy bajo, por lo que no se incluye un análisis comparativo de los resultados pretest/ postest.

Tabla 2: Número y frecuencia de errores morfosintácticos en el pretest y postest

\begin{tabular}{|c|c|c|c|c|c|c|c|c|c|c|c|c|c|c|c|c|c|c|c|c|}
\hline & \multicolumn{20}{|c|}{ Categorías lingüísticas } \\
\hline & \multicolumn{2}{|c|}{$\mathrm{CN}^{\mathrm{a}}$} & \multicolumn{2}{|c|}{ CV } & \multicolumn{2}{|c|}{ TM } & \multicolumn{2}{|c|}{ PR } & \multicolumn{2}{|c|}{ ADV } & \multicolumn{2}{|c|}{$\mathrm{CL}$} & \multicolumn{2}{|c|}{ ADJ } & \multicolumn{2}{|c|}{ SE } & \multicolumn{2}{|c|}{ OT } & \multicolumn{2}{|c|}{ Total } \\
\hline & Pre $^{b}$ & Post & Pre & Post & Pre & Post & Pre & Post & Pre & Post & Pre & Post & Pre & Post & Pre & Post & Pre & Post & Pre & Post \\
\hline Omisión & $6^{c}$ & 6 & 1 & - & 7 & 3 & 2 & 3 & 2 & 1 & 2 & 2 & 1 & 1 & - & 1 & 3 & 2 & 24 & 19 \\
\hline Adición & 1 & - & - & - & 13 & 4 & 12 & 8 & - & 1 & 1 & 3 & 2 & 1 & - & - & - & 1 & 30 & 18 \\
\hline \& Sustitución & 21 & 16 & 3 & 3 & 18 & 17 & 12 & 4 & 1 & - & - & - & 4 & 1 & 3 & 3 & - & - & 62 & 44 \\
\hline$\stackrel{\gtrless}{\&}$ Orden & - & - & - & - & - & 1 & - & - & - & - & - & - & 8 & 4 & - & - & - & 1 & 8 & 6 \\
\hline Combinación & - & - & - & - & - & - & 2 & - & - & 2 & - & - & - & - & - & - & - & - & 2 & 2 \\
\hline Total & 28 & 22 & 4 & 3 & 38 & 25 & 28 & 15 & 3 & 4 & 3 & 5 & 15 & 7 & 3 & 4 & 3 & 4 & 125 & 89 \\
\hline Total $^{\mathrm{d}}(\%)$ & 0,22 & 0,24 & 0,03 & 0,03 & 0,30 & 0,28 & 0,22 & 0,16 & 0,02 & 0,04 & 0,02 & 0,06 & 0,12 & 0,07 & 0,02 & 0,04 & 0,02 & 0,04 & 0,13 & 0,09 \\
\hline
\end{tabular}

La categoría TM incluye los siguientes tipos de errores: sustitución de un tiempo verbal por otro (ejemplo 1) o de un modo por otro (ejemplo 2), la adición de formas nuevas (ejemplo 3) y la omisión de verbos (ejemplo 4):

1. No es completamente de sorpresa que Apple querria traer esa option de Pro a Air standard. (P1/Pretest)

2. (...) pero sería sorprendente de ver que pasa después de unos pocos meses. (P4/Pretest)

3. Apple probablemente introducionar un modelo nuevo del Air. (P6/Pretest)

4. La mas importante es que el cito dice que Apple ha $[X]$ un modelo del Air que trabaja con el Apple Pencil. (P6/Pretest)
Bajo esta categoría, el tipo de error que exhibe la mayor mejoría del pretest al postest es la adición de formas nuevas, es decir, que hubo un número menor de creaciones léxicas ${ }^{6}$ en el postest. La omisión también disminuyó, pero la sustitución de formas se mantuvo prácticamente igual. Los siguientes ejemplos permiten observar la evolución individual de cinco de los seis participantes del estudio que en el pretest recurrieron a creaciones léxicas y que en el postest fueron capaces de emplear formas existentes en español:

5. Apple probablemente introducionar un modelo nuevo del Air. (P6/Pretest)

\footnotetext{
$6 \quad$ Las creaciones léxicas implican una transferencia de forma y significado, y se diferencian de los préstamos en la frecuencia de uso y el nivel de integración social. Mientras que los préstamos suelen emplearse por comunidades de habla, las creaciones léxicas suelen pertenecer a los idiolectos de hablantes individuales (Muñoz Sánchez, 2007).
} 
6. Apple probablemente va a introducir un modelo con mejoras del Air. (P6/Postest)

7. No hay mucho detales a que cuando vamos a expecto para el nuevo IPad moda. (P7/Pretest)

8. No hay muchos detalles sobre a que el nuevo iPad model tiene. (P7/Postest)

9. El sitio Ilame más atención en lo que Apple está expirimentando una versión del Air que funciona con el Apple Pencil. (P5/Pretest)

10. Más importante, dice el sitio que Apple está probando una versión del Air que funciona con el Apple Pencil. (P5/Postest)

11. (...) cuando lo anunica el iPad pro y iPad mini 4. (P3/Pretest)

12. (...) cuando lo anunció el iPad Pro y iPad mini 4. (P3/Postest)

13. (...) pero el cita de "9to5Mac" nos directa a los ideas mas temepranos. (P1/Pretest)

14. (...) pero la cita de "9to5Mac" pone atención a las rumores. (P1/Pretest)

La categoría CN cuenta con casos de sustitución de género (ejemplo 15) o número (ejemplo 16), omisión de determinantes (ejemplo 17) y un caso de adición de determinantes (ejemplo 18):

15. No hay muchas detallas abiertas sobre en el modelo de iPad nuevo. (P1/Pretest)

16. (...) dicen que el Air nuevo puede recibe adicional hablantes y un flash para la camera. (P3/Pretest)

17. (...) que la nueva air podra tener bocinas y $[X]$ flash adicional para su camara. (P4/Pretest)

18. (...) pero el cita de "9to5Mac" nos directa a los ideas mas temepranos. (P1/Pretest)

Bajo esta categoría, el único tipo de error que exhibe una ligera mejoría es la sustitución, lo que parece sugerir un mayor control por parte de los participantes de las categorías de género y número. El caso de adición desaparece en el postest y el número de omisiones se mantiene igual. Los siguientes ejemplos ilustran la evolución individual de algunos de los participantes:
19. Notablemente, la sita dice que Apple ha sido examinar un versión de Air que trabaja con el lápiz Apple. (P3/Pretest)

20. Notable más, el sitio dice que Apple ha sido examinando una versión de el Air que trabajó junto con el Apple Lápiz. (P3/Postest)

21. (...) pero este fall pasada, ha escogido que no aparece el Air 3. (P5/Pretest)

22. (...) pero no sube un Air 3 este otoño pasado. (P5/Postest)

23. No hay mucho informacion sobre expectaciones del iPad modelo nuevo. (P6/Pretest)

24. No hay muchos detalles sobre las expectaciones del nuevo iPad modelo. (P6/Postest)

Es importante destacar que, si bien el número de sustituciones disminuyó ligeramente del pretest al postest, la concordancia de género siguió siendo problemática, lo que se refleja en la presencia de errores de ese tipo durante el segundo periodo de evaluación:

25. (...) mostrando la primera cambia de la linea en mas que un año. (P1/Postest)

26. No hay muchas detalles en lo que expectativas buscar de el nuevo modelo de iPad. (P3/ Postest)

27. (...) que el Air nuevo va a tener sonadores adicionales y una luz para el camera detrás. (P5/Postest)

28. El sitio dice que Apple estaba examinando un version de el Air que trabaja con el Apple Pencil. (P7/Postest)

En la categoría PR se encontraron principalmente ejemplos de sustitución de una preposición por otra y adición de preposiciones innecesarias (ejemplos 29-31). La frecuencia de sustituciones mejora notablemente durante el segundo periodo de evaluación, como se puede apreciar en los siguientes ejemplos (32 y 33):

29. No hay muchas detallas abiertas sobre en el modelo de iPad nuevo. (P1/Pretest)

30. El sitio llame más atención en lo que Apple está expirimentando una versión del Air que funciona con el Apple Pencil. (P5/Pretest) 
31. (...) pero Apple no inducir a el Air 3 en el fall pasado. (P7/Pretest)

32. (...) que muestra el primer cambio en mas que un año de la linea. (P6/Pretest)

33. (...) mostrando el primer cambio de la linea en mas de un ano. (P6/Postest)

En la categoría ADJ se identificaron principalmente casos de orden en los que la posición del adjetivo se ha visto alterada y de sustitución en los que el adjetivo ha sido sustituido por sustantivos. Los casos de sustitución prácticamente desaparecen en el postest, mientras que los de orden disminuyen, pero siguen estando presentes:

34. No hay muchas detalles en como que expectativa de el iPad nuevo modelo. (P3/Pretest)

35. No hay muchas detalles en lo que expectativas buscar de el nuevo modelo de iPad. (P3/ Postest)

36. pero el "9to5Mac" cito refleja chisme. (P6/ Pretest)

37. pero el sito de "9to5Mac" se implica a los rumores tempranos. (P6/Postest)

38. No es una sorpresa que Apple quiere esta misma función del Pro al Air estándar. (P5/ Pretest)

39. No es sorprendente que Apple quiere este funciona en el Air estándar igual que el Pro. (P5/Postest)

En resumen, a pesar del poco tiempo que transcurrió entre el pretest y el postest, el análisis de los datos muestra una ligera disminución de errores morfosintácticos tras el periodo de instrucción, lo que sugiere que la incorporación de la traducción pedagógica tiene un impacto considerable en el desarrollo de la precisión morfosintáctica en la segunda lengua. El aumento de la precisión morfosintáctica es particularmente notable en el uso de preposiciones, adjetivos y el tiempo y modo verbales. Tras el periodo de instrucción, los participantes exhibieron un mayor dominio al seleccionar las preposiciones adecuadas en contextos variados, así como una tendencia menor a incluir preposiciones innecesarias. En cuanto a los adjetivos, tuvieron menos dificultades para posicionarlos en relación con el sustantivo que modifican y para mantener la categoría lingüística de los adjetivos del texto origen en el texto meta, es decir, para traducir los adjetivos como adjetivos sin tener que sustituirlos por sustantivos. Por último, en cuanto al tiempo y modo verbales, demostraron una mayor habilidad para usar formas verbales existentes en la lengua sin tener que recurrir a la creación léxica.

La prevalencia de errores morfosintácticos en el pretest no debería sorprender, ya que -como han señalado otros autores- en la traducción, a diferencia de la escritura libre, los estudiantes no pueden recurrir a lo que González Davies (2002) denomina estrategias de evasión para evitar los ítems lingüísticos que les resultan problemáticos o les crean inseguridades.

\section{CONCLUSIONES E IMPLICACIONES PEDAGÓGICAS}

La frecuencia menor de errores morfosintácticos tras el periodo de instrucción sugiere que la incorporación de la traducción pedagógica en clases de nivel avanzado mediante una metodología activa que combina el trabajo individual y colaborativo, y promueve la reflexión tiene un impacto considerable en la precisión morfosintáctica en la segunda lengua. Este impacto positivo se hace particularmente notable -como se ha visto- en el uso de preposiciones, adjetivos y el tiempo y modo verbales.

Los resultados de este estudio contradicen las afirmaciones de Lado (1964) y Gatenby (1967) sobre las consecuencias negativas de la práctica de la traducción en la precisión gramatical, por lo que se suma al trabajo de autores como Cook (2010) y González Davies (2002), que proponen la práctica de la traducción inversa ( $\mathrm{L} 1>\mathrm{L} 2)$ para desarrollar la precisión gramatical.

Por último, como han señalado otros investigadores previamente (Carreres y Noriega-Sánchez, 2011; Cummins, 2007; Hurtado Albir, 2001 y KäIlkvist, 2008), conviene subrayar la importancia de realizar más investigaciones empíricas que evalúen los efectos de la traducción pedagógica. 


\section{LIMITACIONES}

Si bien este estudio preliminar ofrece resultados de sumo interés, presenta un número de limitaciones: (i) el número de participantes fue limitado, ya que dependía de la cantidad de estudiantes matriculados en el curso; (ii) no se incluyó un grupo de control; (iii) no fue posible llevar a cabo un segundo postest, ya que la mayoría de los estudiantes se graduaron y abandonaron la institución al terminar el curso.

\section{REFERENCIAS BIBLIOGRÁFICAS}

Belpoliti, F. y Plascencia-Vela, A. (2013). Translation techniques in the spanish for heritage learners' classroom: Promoting lexical development. En D. Tsagari (Ed.), Translation in language teaching and assessment (pp. 6591). Newcastle: Cambridge Scholars Publishing.

Carreres, Á. y Noriega-Sánchez, M. (2011). Translation in language teaching: Insights from professional translator training. The Language Learning Journal, 39(3), 281-297. doi:10.108 0/09571736.2011.567356

Cohen, A. D. y Brooks-Carson, A. (2001). Research on direct versus translated writing: Students' strategies and their results. The Modern Language Journal, 85(2), 169-188.

Colina, S. (2002). Second language acquisition, language teaching and translation studies. The Translator, 8(1), 1-24. doi:10.1080/1355 6509.2002.10799114

Cook, G. (2010). Translation in language teaching: An argument for reassessment. Oxford: Oxford University Press.

Cummins, J. (2007). Rethinking monolingual instructional strategies in multilingual classrooms. Canadian Journal of Applied Linguistics/Revue canadienne de linguistique appliquée, 10(2), 221-240.

Cummins, J. (2008). Teaching for transfer: Challenging the two solitudes assumption in bilingual education. En N. Hornberger (Ed.), Encyclopedia of language and education (pp. 65-77). New York: Springer Science.
Duff, A. (1989). Translation. Oxford: Oxford University Press.

Fairclough, M. y Mrak, A. (2003). La enseñanza del español a los hispanohablantes bilingües y su efecto en la producción oral. En A. Roca y C. Colombi (Eds.), Mi lengua: Spanish as a heritage in the United States, research and practice (pp. 198-212). Washington D.C.: Georgetown UP.

Gasca Jiménez, L. (en prensa). Las posibilidades de la traducción pedagógica para la enseñanza del español como segunda lengua. Estudios de Lingüística Aplicada, 66.

Gatenby, E. V. (1967). Popular fallacies in the teaching of foreign languages. ELT Journal, 7(1), 21-29.

González Davies, M. (2002). Translation in foreign language learning: Sleeping with the enemy? APAC of news, 64-74.

González Davies, M. (2004). Multiple voices in the translation classroom: Activities, tasks and projects. Amsterdam: J. Benjamins.

Harvey, M. (1996). A translation course for frenchspeaking students. En P. Sewell y I. Higgins (Eds.), Teaching translation in universities: Present and future perspectives (pp. 15-30). Londres: Middlesex University Printing Services.

Hurtado Albir, A. (2001). Traducción y traductología: Introducción a la traductología. Madrid: Cátedra.

James, C. (1998). Errors in language learning and use: Exploring error analysis. New York: Routledge.

Källkvist, M. (1996). How different are the results of translation tasks? A study of lexical errors. En K. Malmkjær (Ed.), Translation and language teaching (pp. 77-89). Manchester: St. Jerome Publishing.

Källkvist, M. (2004). The effect of translation exercises versus gap-exercises on the learning of difficult I2 structures. En K. Malmkjær (Ed.), Translation in undergraduate degree programmes (Vol. 59, pp. 163-184). Amsterdam/ Philadelphia: John Benjamins Publishing Company. 
El efecto de la traducción pedagógica en la precisión morfosintáctica: un estudio preliminar con estudiantes avanzados de ESL

Källkvist, M. (2008). L1-I2 translation versus no translation: A longitudinal study of focus-onforms within a meaning-focused curriculum. En L. B. Ortega, Heidi (Ed.), The longitudinal study of advanced I2 capacities (pp. 182202). New York: Routledge.

Kiraly, D. (2000). A social constructivist approach to translator education: Empowerment from theory to practice. Manchester: St. Jerome Pub.

Kobayashi, H. y Rinnert, C. (1992). Effects of first language on second language writing: Translation versus direct composition. Language Learning, 42(2), 183-209.

Lado, R. (1964). Language teaching. A scientific approach. New York: McGraw-Hill.

Laufer, B. y Girsai, N. (2008). Form-focused instruction in second language vocabulary learning: A case for contrastive analysis and translation. Applied Linguistics, 29(4), 694-716. doi:10.1093/applin/amn018

Malmkjær, K. (1998). Translation and language teaching: Language teaching and translation. Manchester: St. Jerome Publishing.

Mellinger, C. D. y Hanson, T. A. (2017). Quantitative research methods in translation and interpreting studies. New York: Routledge.

Muñoz Sánchez, A. (2007). Register and style variation in speakers of spanish as a heritage and as a second language (Tesis doctoral). Recuperada de la base de datos de ProQuest Dissertations and Theses. (AAT 3262717).

Richards, J. y Rodgers, T. (2014). Approaches and Methods in Language Teaching. UK: Cambridge University Press.

Sánchez Cuadrado, A. (2016). Aprendizaje formal de ele mediante actividades cooperativas de traducción pedagógica con atención a la forma (Tesis doctoral, Universidad de Granada, Granada, España). Recuperada de http://hdl. handle.net/10481/41765.

Schjoldager, A. (2004). Are I2 learners more prone to err when they translate. En K. Malmkjær (Ed.), Translation in undergraduate degree programmes (pp. 127-152). Philadelphia: John Benjamins Publishing Company.

Snell-Hornby, M. (1985). Translation as a means of integrating language teaching and linguistics. En C. H. Titford y A. E. Hieke (Eds.), Translation in foreign language teaching and testing (pp. 21-28). Germany: Gunter Narr Verlag Tübingen.

Snellings, P., Van Gelderen, A. y De Glopper, K. (2002). Lexical retrieval: An aspect of fluent second-language production that can be enhanced. Language Learning, 52(4), 723-754.

Sørensen, K. (1990). Translation as a unifying discipline. En S. Larsen (Ed.), Translation. A means to an end (pp. 57-68). Aarhus: Aarhus University Press.

Titford, C. (1985). Translation - a post-communicative activity for advanced learners. En C. $\mathrm{H}$. Titford y A. E. Hieke (Eds.), Translation in foreign language teaching and testing (pp. 73-86). Germany: Gunter Narr Verlag Tübingen.

Vaezi, S. y Mirzaei, M. (2007). The effect of using translation from I1 to $\mathrm{I} 2$ as a teaching technique on the improvement of efl learners' linguistic accuracy-focus on form. Humanising Language Teaching, 9(5).

\section{ANEXO A}

\section{Ejemplo de tarea}

Este fragmento proviene de un folleto para promocionar la Universidad de Houston. La traducción estará dirigida a estudiantes de la Universidad de Cádiz que estén interesados en estudiar un año de su licenciatura en los Estados Unidos. El folleto será distribuido durante sesiones informativas en la Universidad de Cádiz.

\section{UNIVERSITY OF HOUSTON}

Founded in 1927, the University of Houston is the leading public research university in the vibrant international city of Houston. Each year, we educate more than 40,750 students in more than 300 undergraduate and graduate academic programs, on campus and online. UH awards more than 
8,000 degrees annually, with more than 230,000 alumni.

\section{Location}

$\mathrm{UH}$ is located in Houston, Texas, the nation's fourth-largest city and the energy capital of the world. Our students regularly test their skills through internships with national and international companies based here, and our faculty routinely partner with businesses and government agencies through research.

Programs of Study

Our undergraduates choose from 120 majors and minors. At the graduate level, we offer 139 master's, 54 doctoral, and three professional degree programs. You may study online through our Distance Education program, or take noncredit courses through Continuing Education. Many of our academic programs rank among the nation's best.

\section{Research}

Our faculty and students conduct research through 25 research centers and in every academic department. UH research regularly breaks new ground and opens doors to new ways of understanding the world.

\section{Ejemplo de entrega}

\section{LA UNIVERSIDAD DE HOUSTON}

La Universidad de Houston fue fundada en 1927, la cual es la universidad pública de investigaciones más destacada de la ciudad internacional y diverso de Houston. Cada año, educamos más que 40.750 estudiantes en más que 300 programas académicos grados y postgrados, los cuales ofrecen clases presenciales y en línea. La Universidad de Houston concede más que 8.000 licenciaturas cada año y tiene más que 230.000 alumnos graduados.

\section{Ubicación}

La Universidad de Houston está situada en Houston, Texas- la cuarta ciudad más grande en los Estados Unidos y el centro de energía del mundo. Nuestros estudiantes usan sus habilidades mediante prácticas con las compañías nacionales e internacionales cuales son localizados en Houston; también, nuestro profesorado se asocian con las compañías y agencias gubernamentales regularmente por medio de la investigación.

\section{Programas Académicos}

Para los programas grados, hay 120 especializaciones y subespecializaciones que podáis elegir. De postgrado, tenemos 137 másteres, 54 doctorados, y 3 profesional programas académicos. Podáis estudiar en línea a través de nuestra programa Distance Education (cursos no en persona), o podáis tomar cursos sin créditos a través de Continuing Education (cursos de enseñanza para adultos). Muchos de nuestros programas académicos están calificados con los mejores de los Estados Unidos.

\section{Las Investigaciones}

Nuestro profesorado y los estudiantes hacen investigaciones con 25 centros de investigaciones y en cada facultad académica. Los investigaciones de la Universidad de Houston son revolucionarios así abren nuevos caminos para entender el mundo de maneras nuevas y diferentes.

\section{Comentario de traducción:}

La función del texto meta es promocionar la Universidad de Houston. La audiencia es los estudiantes de la Universidad de Cádiz (que estén interesados en estudiar un año de su licenciatura en los Estados Unidos). La modalidad es un folleto. Estará disponible durante sesiones informativas en la Universidad de Cádiz.

Retos y estrategias:

Las dificultades que me enfrenté eran la terminología de escuela porque hay ideas diferentes de educación en los Estados Unidos versus las de España, así hay términos diferentes de cada uno, por lo tanto tenía que buscar cuales debo usar y cómo las Ilaman en España. Más de eso, las metáforas requieren más tiempo para buscar cómo debe decir la misma idea en la otra lengua.

Para solucionar los problemas que me enfrenté, he usado SpanishDict, WordReference, y ReversoContext, cuales son sitios de web que me ayudan mucho para encontrar definiciones, frases, 
El efecto de la traducción pedagógica en la precisión morfosintáctica: un estudio preliminar con estudiantes avanzados de ESL

sinónimos, y ejemplos de palabras y también muestran éstas dentro de un contexto real. Creo que he usado casi todas las técnicas de traducción sin darme cuenta, pero específicamente he usado:

- Traducción literal: "Each year, we educate more than 40,750 students" à "Cada año, educamos más que 40.750 estudiantes"

- Transposición: "is the leading public research university" à "es la universidad pública de investigaciones más destacada"

- Modulación: "breaks new ground" à "abren nuevos caminos"

- Adaptación: "undergraduate programs" à "programas académicos grados"

- Compensación: "new ways of understanding the world" à "entender el mundo de maneras nuevas y diferentes"

- Explicitación: "Distance Education" à "Distance Education (cursos no en persona)"

\section{ANEXO B}

\section{Pretest/ postest}

\section{NEW IPAD AIR}

A new iPad Air appears to be on the way. According to some sources, Apple is likely to introduce an updated model of the Air during an event in mid-March, marking the line's first change in over a year. The Air 2 was released in October 2014, but Apple chose not to introduce an Air 3 this past fall, when it announced the iPad Pro and iPad Mini 4. There aren't many details on what to expect from the new iPad model, but the "9to5Mac" site points to earlier rumors saying the new Air may get additional speakers and a flash for its rear camera. Most notably, the site says that Apple has been testing a version of the Air that works with the Apple Pencil. It's not entirely surprising that Apple would want to bring that feature from the Pro to the standard Air, but it would be surprising to see that happen after just a few months. 